\title{
Practical Gains from Access to an Integrated Disease Reporting System
}

\author{
Loren Shaffer* \\ Northrop Grumman Information Systems, Atlanta, GA, USA
}

\section{Objective}

We describe an electronic disease reporting system that integrates case-based disease information from humans and other animals in a single database and examine the utility for supporting disease surveillance functions through access to longitudinal case reports of multiple diseases across multiple species provided by the system.

\section{Introduction}

The Electronic Integrated Disease Surveillance System (EIDSS) is a computer-based disease reporting application funded under the Cooperative Biological Engagement Program of the U.S. Defense Threat Reduction Agency. EIDSS deployment includes the Republics of Georgia (GG) and Azerbaijan (AJ) where personnel in the Ministry of Health and the Ministry of Agriculture in each country enter casebased disease reports.

The potential benefits obtained through surveillance of infectious diseases across species have been widely discussed. A limitation of such practice has been the paucity of single applications that collect information about disease in both human and other animal populations (Scotch 2009). A unique feature of EIDSS is the use of a single platform to enter reports of disease in humans and other animals. Records are stored in a common database enabling ready access to information on multiple diseases and provide a quantitative linkage between human and animal data. An integrated analysis and reporting (AVR) module further supports timely investigation of disease events across the epizootic barrier.

\section{Methods}

EIDSS records of cases diagnosed beginning 1 Jan 2010 through 5 Nov 2011 (GG) and 1 Jan 2011 through 27 Mar 2012 (AJ) were examined to assess timeliness of entry, completeness and disease representation. Records corresponding to a known outbreak of anthrax (GG) and occurrence of brucellosis (AJ) were examined using methods available in the AVR module of EIDSS to determine incidence, prevalence and occurrence. Results were then evaluated for utility as a public health practitioner might use them.

\section{Results}

Significantly increased reports of anthrax in humans and livestock in Kvemo-Kartli Georgia, as determined by CuSum analysis, occurred 20 days before an outbreak of cutaneous anthrax was announced. Out of 201 total human anthrax cases, only one was reported as gastrointestinal. However, significant increases of human diarrheal disease reports occurred in temporal proximity to this outbreak within the same area. Gastrointestinal anthrax has been demonstrated to present with diarrhea in $80 \%$ of cases in humans (Ndyabahinduka 1984).

In Azerbaijan brucellosis repots comprise $2.1 \%$ of all reports in humans and $51.2 \%$ in livestock. A previously unidentified clustering of brucellosis reports in humans was discovered in Shemkir. National incidence of brucellosis was determined to be 4.1 per 100,000 . In Shemkir incidence was 19.1 per 100,000 . Three areas adjacent to Shemkir were discovered to have elevated prevalence of brucellosis in livestock.

\section{Conclusions}

Timely access to disease data for multiple species may better enable agencies responsible for public health to characterize and understand disease events. In some instances perhaps identifying cases that might otherwise go unrecognized. EIDSS is one of the first solutions in public health practice to provide such unique access by collecting records in a single database that is accessible through a common user interface. As the technical core for a national disease reporting system in many countries of the former Soviet Union, EIDSS supports agencies involved in the investigation of zoonotic diseases and outbreaks that may occur. The practical benefits of such integration and accessibility, demonstrated by the examination of records as described, provide an added measure of timeliness of potential discovery in addition to a comprehensive understanding of disease events in terms of epidemiology and impact.

\section{Keywords}

Zoonoses; Disease Surveillance; Outbreak Detection; One-health; Cluster Detection

\section{References}

Scotch M, Odofin L, Rabinowitz P. Linkages between animal and human health sentinel data. BMC Veterinary Research 2009;5(15).

Ndyabahinduka, D., I. Chu, et al. An outbreak of human gastrointestinal anthrax. Ann 1st Super Sanita 1984;20(2-3): 205-208.

\author{
*Loren Shaffer \\ E-mail: Loren.Shaffer@ngc.com
}

